\title{
An isogeometric analysis approach for two- dimensional steady state heat transfer problems
}

- Le Tuan Em

- Nguyen Duy Khuong

- Vu Cong Hoa

Ho Chi Minh city University of Technology, VNU-HCM

(Manuscript Received on August 01 ${ }^{s t}$, 2015, Manuscript Revised August 27 ${ }^{\text {th }}$, 2015)

\section{ABSTRACT:}

The purpose of this article is studied the application of isogeometric analysis (IGA) to two-dimensional steady state heat transfer problems in a heat sink. By using high order basis functions, NURBS basis functions, IGA is a high rate

Key words: iga, heat transfer, nurbs

\section{INTRODUCTION}

Almost every technical operate process generate heat during activity duration; it can be active or inactive. In case of parts work in high temperature conditions, the size, material and other relative parameters must be optimized so that they can avoid destroying, and heat which generate unnecessary needed to effectively diffused [1,2]. It is an importance and necessity for heat transfer problems in techniques and industries, and it is interested in science and engineering communities. The heat transfer problems have solved by many different methods, like Finite Element Method [3], meshless method [4], or Finite Pointset Method for simulating heat transfer involving a moving source [5], even Analytical Solution. In this study, we focus on the introduction of the basic concept of isogeometric analysis using B-spline basis functions for heat transfer problems and discuss the accuracy of this method also mentioned other. convergence approach in comparison to a traditional Finite Element Method. Moreover, the development of this method decreased the gaps between $C A D$ and mathematical model and increased the continuity of mesh.

Isogeometric analysis (IGA) was introduced in [6] and has developed since 2005. Because of the existing gaps between Computer Aided Design (CAD) and the Finite Element Analysis (FEA), IGA was coined. The predominance that is using No-Uniform Rational B-spline to represent the complex geometries, while the geometry is replaced by finite element meshes approximated of the geometry in FEA. To obtain a high accuracy result, a refinement mesh is used with a coherence level. In traditional FEA, the refinement requires communication with the CAD geometry during a process of analysis, while simplify mesh refinement is a dominance of IGA. It is approximately $80 \%$ of overall analysis time to generate the mesh in FEM [7]. Therefore, we will save much time and cost with IGA. The IGA has been applied to several physical problems and will be clearly described in this paper.

\section{BSPLINE AND NURBS}

\section{Trang 164}




\subsection{Knot vectors}

A knot vector is a set of knots which are defined in the parameter space of a curve, that is

$$
\Xi=\left\{\xi_{1}, \xi_{2}, \ldots, \xi_{n+p+1}\right\}
$$

where $\xi_{i} \in \square$ is the $i^{\text {th }}$ knot, $i$ is the knot index, $i=1,2, \ldots, n+p+1, \quad p$ is the polynomial order, and $n$ is the number of basis functions used to contruct the B-spline curve. If the knots are equally spaced in the parameter space, knot vectors may be uniform.

\subsection{Basis functions}

The B-spline basis functions are recursive functions [8]. For $p=0$, they are defined by

$$
N_{i, 0}(\xi)=\left\{\begin{array}{ccc}
1 & \text { if } & \xi_{i} \leq \xi<\xi_{i+1} \\
0 & \text { otherwise }
\end{array}\right.
$$

and for $p=1,2,3, \ldots$

$$
\begin{aligned}
N_{i, p}(\xi)= & \frac{\xi-\xi_{i}}{\xi_{i+p}-\xi_{i}} N_{i, p-1}(\xi) \\
& +\frac{\xi_{i+p+1}-\xi}{\xi_{i+p+1}-\xi_{i+1}} N_{i+1, p-1}(\xi)
\end{aligned}
$$

where $1 \leq i \leq n$ and $0 / 0$ is considered as zero.

Clearly, considering a B-spline basis function with $p$ degree, the interior knot can be a multiplicity $p$ in the knot vector. Furthermore, the first and last knots have multiplicity $p+1$ that is open knot vector. Major properties of the B-spline basis functions involve non-negativity, partition of unity, local support and $C^{p-k}$ continuty. An example of open knot vector $\Xi=\{0,0,0,0.25,0.5,0.75,0.75,1,1,1\}$ is presented in Figure 1.

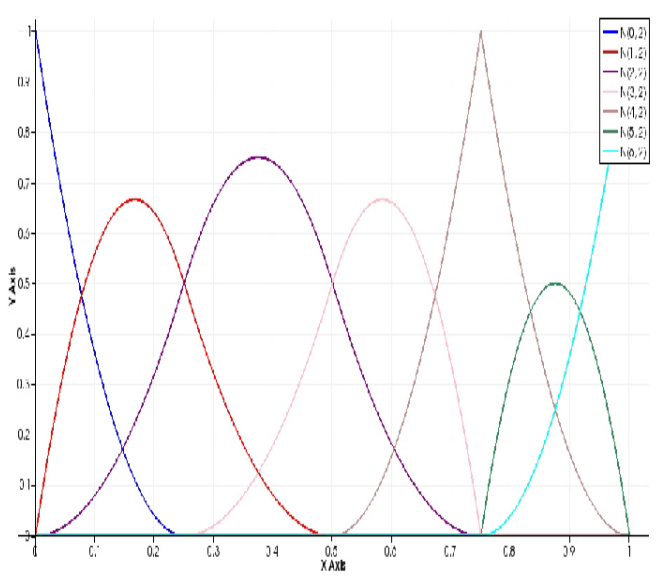

Figure 6. Quadratic basis functions for the open knot vector $\Xi=\{0,0,0,0.25,0.5,0.75,0.75,1,1,1\}$

There are several important features of NURBS geometry. The first is that the basis constitutes a partition of unity, that is

$\sum_{i=1}^{n} N_{i, p}(\xi)=1 \quad \forall \xi$

The second, each basis function is pointwise nonnegative over the entire domain, that is $N_{i, p}(\xi) \geq 0$. The next feature is that each $p^{\text {th }}$ order function has $p-1$ continuous derivatives across the element boundary. And an important note is the support of the B-spline functions of order $p$ is always $p+1$ knot spans.

\subsection{B-spline curves}

A B-spline curve for a given direction has the form of

$\mathbf{C}(u)=\sum_{i=0}^{n} N_{i, p}(u) w_{i} \mathbf{P}_{i}$

NURBS are B-spline generalization and alow more control over local domain

$\mathbf{C}(u)=\frac{\sum_{i=0}^{n} N_{i, p}(u) w_{i} \mathbf{P}_{i}}{\sum_{i=0}^{n} N_{i, p}(u) w_{i}} \quad a \leq u \leq b$ 
where the $\mathbf{P}_{i}$ are the control points, the $w_{i}$ are the weights, and the $N_{i, u}(u)$ are the pth-order B-spline basis functions defined on the nonperiodic (and nonuniform) knot vector

$U=\left\{a, \ldots, a, u_{p+1}, \ldots, u_{m-p-1}, b, \ldots, b\right\}$

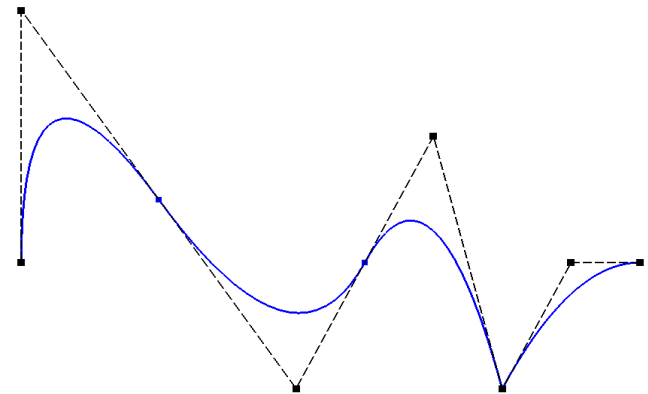

Figure 7. B-spline, piecewise quadratic curve in $\square$. Basis function and knot vector as in Figure 1. Control point locations are denoted by $\mathbf{\square}$, and the knots, which define a mesh by partitioning the curve into elements, are denoted by

\subsection{B-spline surfaces}

Given a control net $\left\{B_{i, j}\right\}, i=1,2, \ldots, n$, $j=1,2, \ldots, m$, knot vectors $\Xi=\left\{\xi_{1}, \xi_{2}, \ldots, \xi_{n+p+1}\right\}$ and $\mathrm{H}=\left\{\eta_{1}, \eta_{2}, \ldots, \eta_{m+q+1}\right\}$, a tensor product $\mathrm{B}$ spline surface is defined by

$$
S(\xi, \eta)=\sum_{i=1}^{n} \sum_{j=1}^{m} N_{i, p}(\xi) M_{j, p}(\eta) \mathbf{B}_{i, j}
$$

where $N_{i, p}(\xi)$ and $M_{j, p}(\eta)$ are univariate B-spline basis functions of order $p$ and $q$, corresponding to knot vector $\Xi$ and $H$, respectively.

\section{A TWO-DIMENSIONAL STATIC FORMULATION BASED ON NURBS APPROXIMATIONS}

Using NURBS basis function, the temperature variable can be interpolated as

$$
\mathbf{T}=\sum_{i=1}^{n} R_{i} \mathbf{T}_{i}
$$

where $R_{i}$ are the NURBS basis functions, $\mathbf{T}_{i}$ are the temperature at control point (7) $i$ and $n$ is the number of control points.

The governing equation of static analysis for a linear structural system in the form as

$$
\mathbf{K T}=\mathbf{f}
$$

where $\mathbf{K}$ is the global left hand side matrix expressing the properties of the overall system, $\mathbf{f}$ is the global load vector, which is the assemblage of individual load vectors.

In addition, $\mathbf{K}$ matrix is presented by [9]

$$
\mathbf{K}=\int_{\Omega} \mathbf{B}^{T} \mathbf{D B} d \Omega+\int_{\Gamma} h \mathbf{N}^{T} \mathbf{N} d \Gamma
$$

where $\mathbf{B}$ is the derivative matrix, which relate the gradient of the field variable to the nodal values. And $\mathbf{D}$ matrix in form as

$$
\mathbf{D}=\left[\begin{array}{cc}
k_{x} & 0 \\
0 & k_{y}
\end{array}\right]
$$

where $k_{x}$ and $k_{y}$ is the thermal conductivity coefficients.

\section{RESULTS AND DISCUSSION \\ 4.1 Square plate with Dirichlet conditions}

To demonstrate the accuracy and performance of the isogeometric in heat conduction problems, we consider to a square plate of unit thickness, is shown in Figure 8, size $100 \mathrm{~cm}$. At the top side, the plate is subjected to isothermal boundary conditions of $500^{\circ} \mathrm{C}$, and $100^{\circ} \mathrm{C}$ at the other sides [9, 10]. Assume the thermal conductivity of the material is constant and equal to $10 \mathrm{~W} / \mathrm{m}^{\circ} \mathrm{C}$. Determine the temperature at the center of the plate using 
Isogeometric Analysis and compare that result with the analytical method and the Finite Element Method.

In order to obtain the exact solution of the steady state without heat generator, we shall use the Laplace equation and the analytical solution was calculated by Holman, 1989, [6], it is a Fourier sine series, and that solution is expressed in Equation (13).

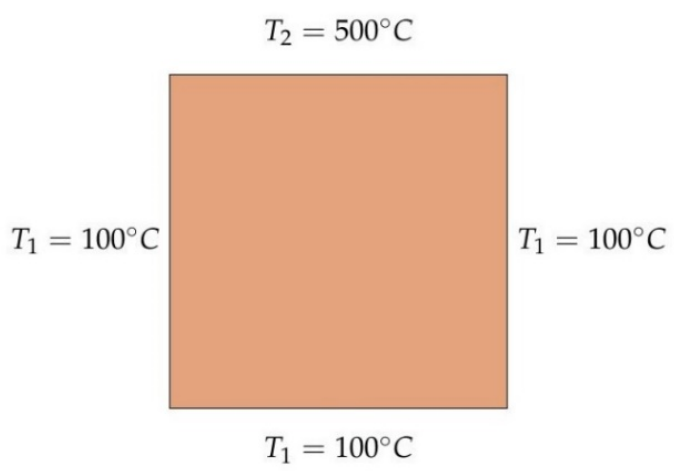

Figure 8. Square plate with boundary conditions

$$
\begin{aligned}
T- & T_{1}=\left(T-T_{2}\right) \\
& \times \frac{2}{\pi} \sum_{n=1}^{\infty} \frac{(-1)^{n+1}+1}{n} \sin \frac{n \pi x}{W} \frac{\sinh (n \pi y / W)}{\sinh (n \pi H / W)}
\end{aligned}
$$

where, $T_{2}$ is the temperature at the top side, and $T_{1}$ is at the other sides.

By apply the concrete boundary conditions and a range of $n$ value, the temperature at the center of the plate is determined following the Equation (13). The value of temperature is static at $T_{\text {center }}=200.000^{\circ} \mathrm{C}$.

First, we consider to the basis is choosen as quadratic, cubic and quartic NURBS, is shown in Figure 9. The number of elements is constant while the number of control points and the order of basis functions simultaneously increase. To clearly observe the advantage of IGA, we consider to the temperature in the center of square plate with array of degree of freedoms.

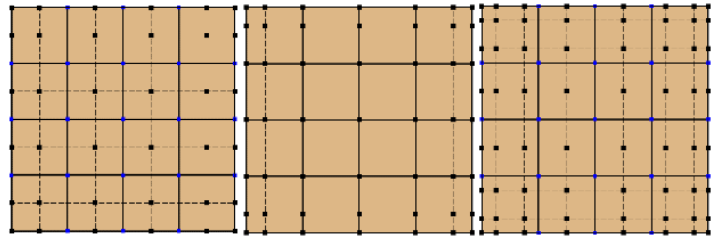

Figure 9. Quadratic, cubic and quartic mesh

The convergence of the temperature at the center of square plate is show in the Figure 10. According to the figure, by increasing the order, $p$, of the basis functions, the results show higher accuracy.

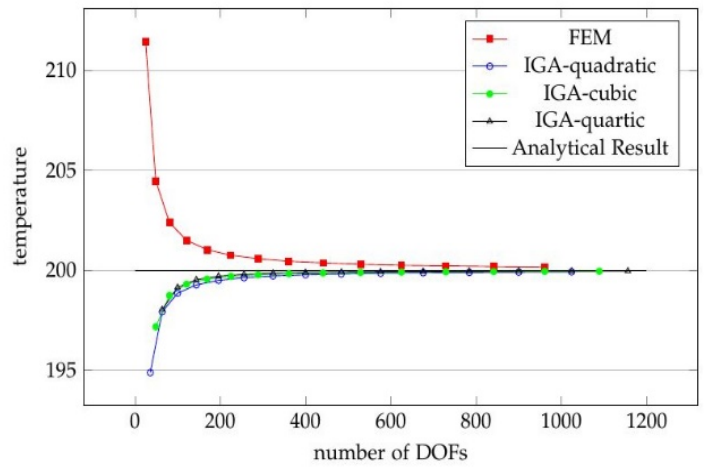

Figure 10. The comparisons of convergence of the temperature at the center of square plate

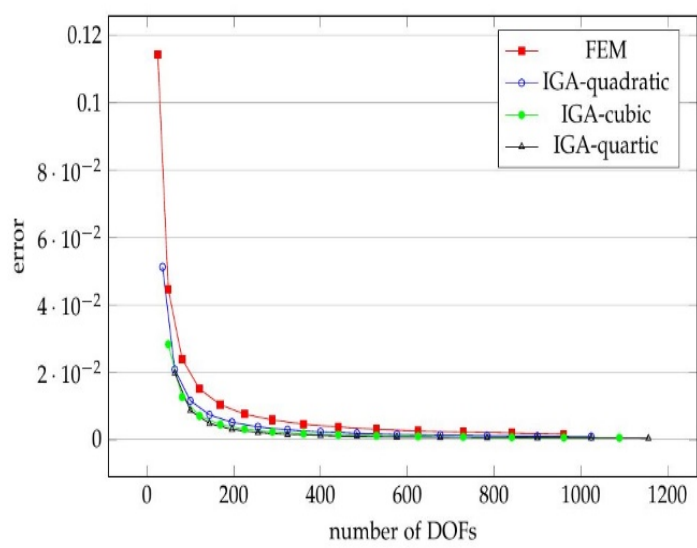

Figure 11. The error value to the analytical solution of each case

Although, the cubic and quartic IGA gain uper-convergence with a very small error, about $0.008-0.433 \%$. By increasing the order, $p$, of the basis functions, the obtained results converge to the best reference value determined by the expression (13), [7]. By using more fine 
discretization, there errors are reduces, as shown in Figure 11.

As shown in the Figure 10, the convergence rate of the FEA is slower than IGA, it mean, at the certain number of DOFs, the IGA result closer the analytical result than FEA. The temperature distribution in the square plate is shown in Figure 12.
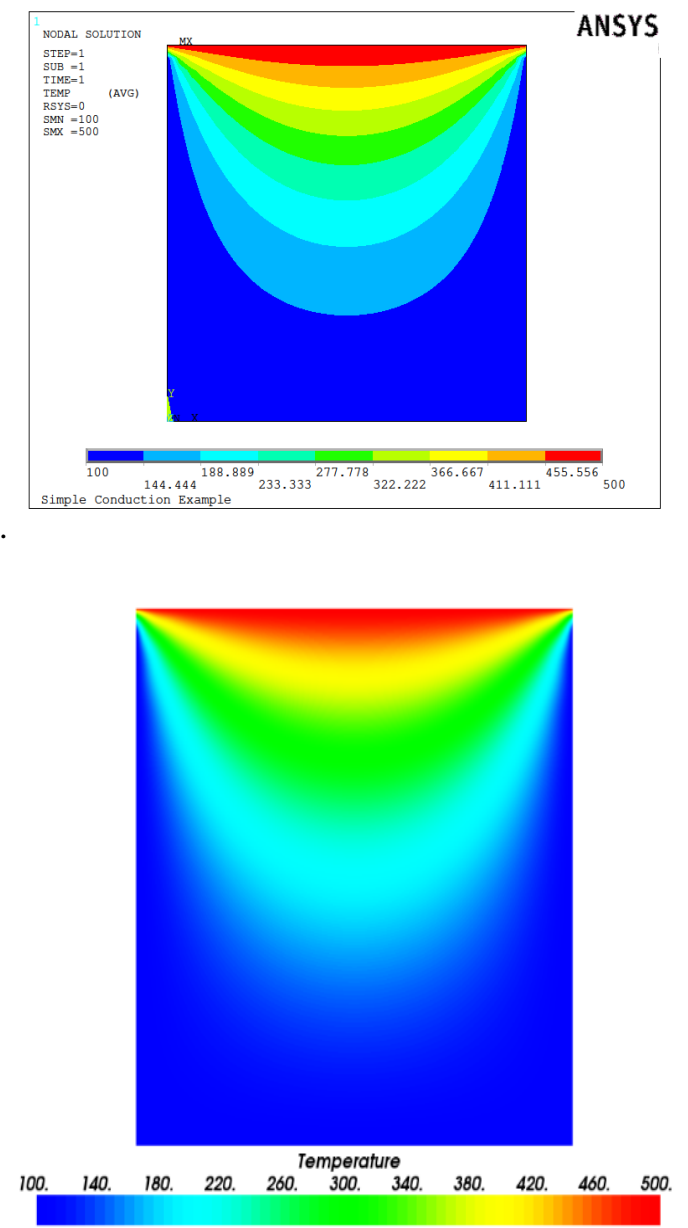

Figure 12. The temperature distribution with 961 DOFs in FEM and 361 DOFs in IGA

\subsection{Square plate with both Dirichlet and Neumann conditions}

As a second example, a two-dimensional domain is prescribed with Dirichlet and Neumann boundary conditions applied along the boundaries is show in Figure 13. Heat enter at the bottom of the plate is $500^{\circ} \mathrm{C}$, and other sides entered Neumann conditions with the convection heat transfer coefficient $h=10 \mathrm{~W} / \mathrm{m}^{2} \mathrm{~K}$ and the temperature of the fluid is $T_{f}=100^{\circ} \mathrm{C}$.

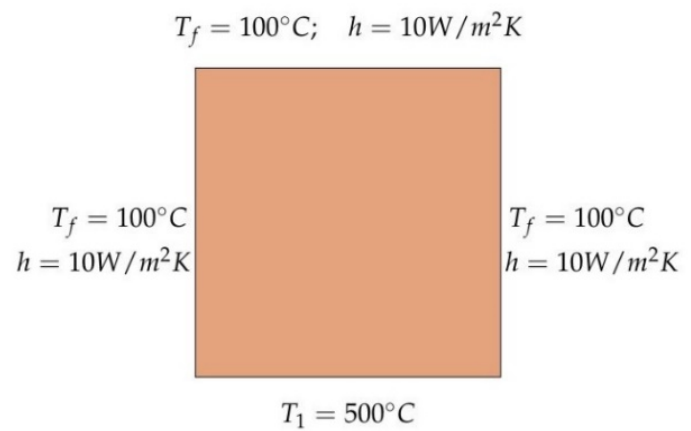

Figure 13. Square plate with both Dirichlet and Neumann boundary conditions

The lowest temperature is at the top-left and right corner, $T=203^{\circ} \mathrm{C}$ and the highest temperature certainly is at the bottom of square plate. The plot of temperature distribution is shown in the Figure 14.

The

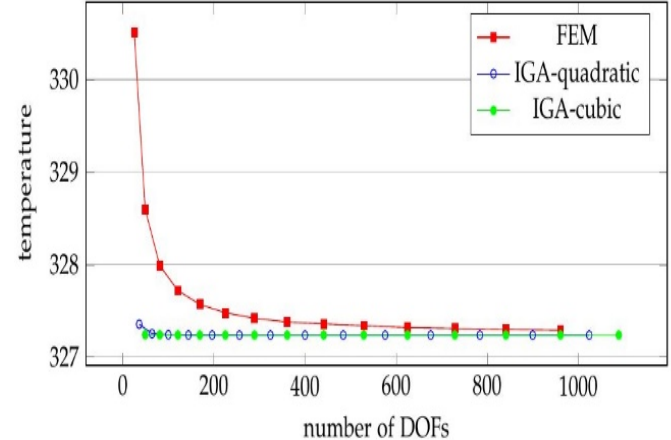

Figure 15 shows the convergence of the temperature at the center of the square plate. As increasing a number of control points, the obtained results converge to a value.

Both of two method have similar temperature distribution in this problem, it is shown in Figure 16. 

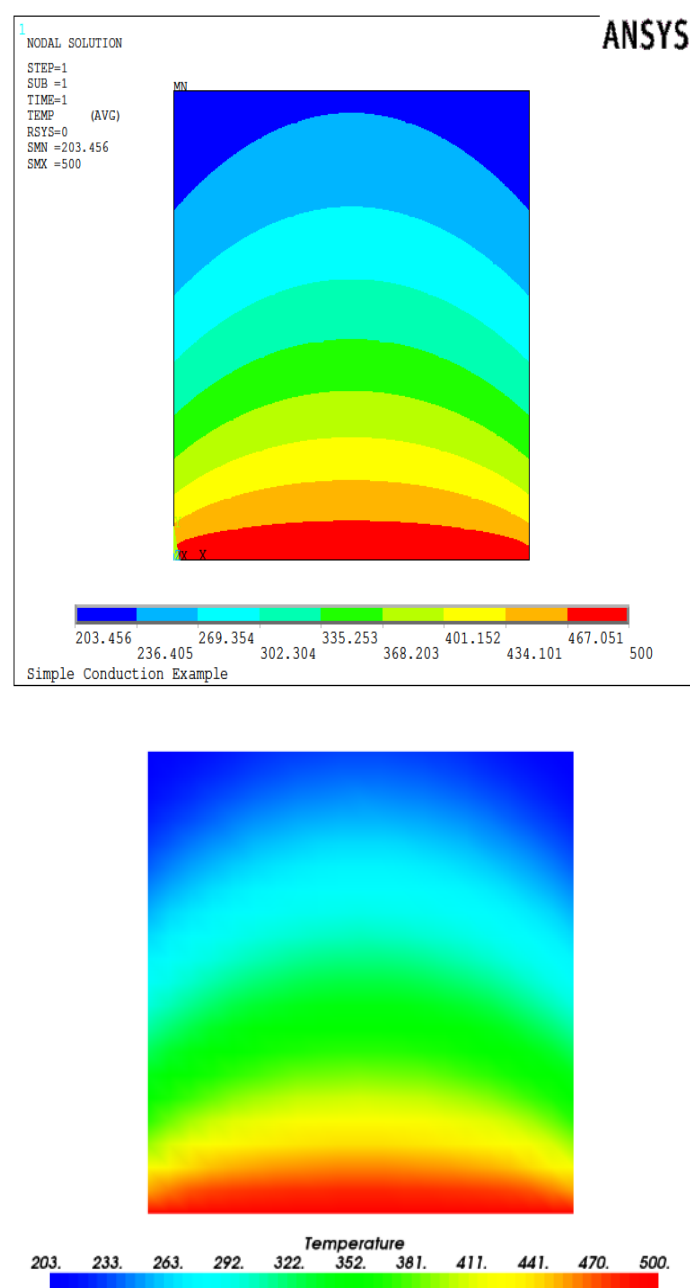

Figure 14. The temperature distribution in the square plate with 961 DOFs base on FEM and 49

DOFs base on cubic IGA

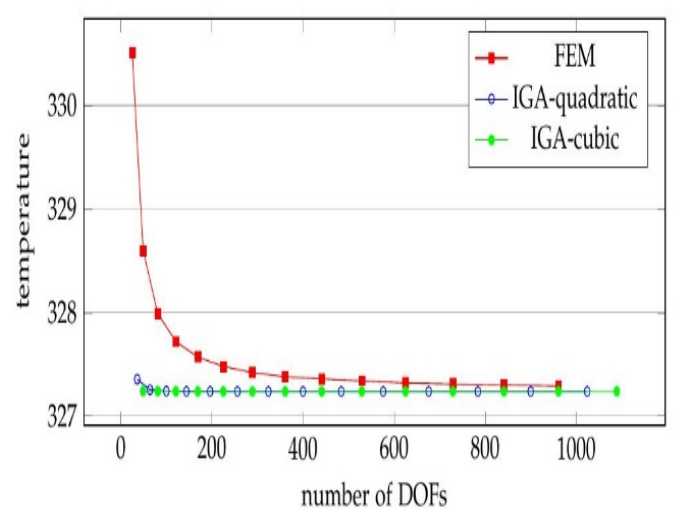

Figure 15. The comparisons of convergence of the temperature at the center of square plate with component of Neumann and Dirichlet Conditions

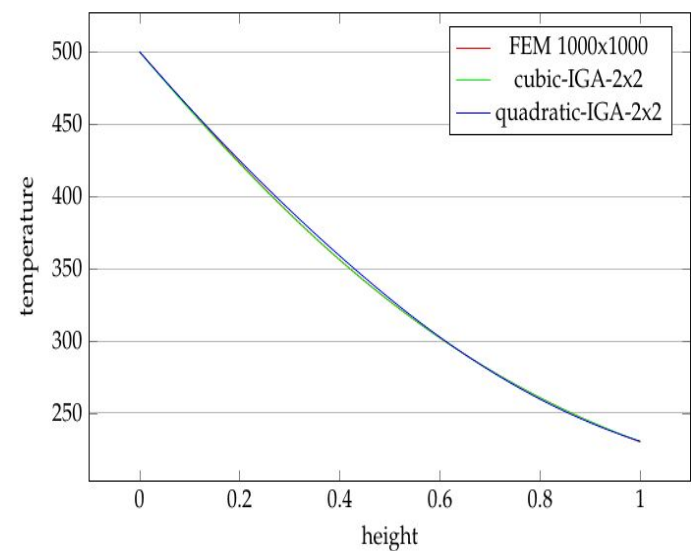

Figure 16. The temperature distribution on $y$ direction

\subsection{A quarter of annular}

In order to gain a better understanding of effect of IGA, we focus on a problems with circle boundaries, as show in Figure 17. It is a quarter of annular with $r_{1}=0.1(\mathrm{~m})$ and $r_{2}=0.2(\mathrm{~m})$. The boundaries conditions is similar to square plate with Dirichlet and Neumann conditions as expressed in the above: the temperature value at the bottom is $500^{\circ} \mathrm{C}$ and the convection conditions on other sides with the convection heat transfer coefficient $h=10 \mathrm{~W} / \mathrm{m}^{2} \mathrm{~K}$ and the temperature of the fluid is $T_{f}=100^{\circ} \mathrm{C}$.

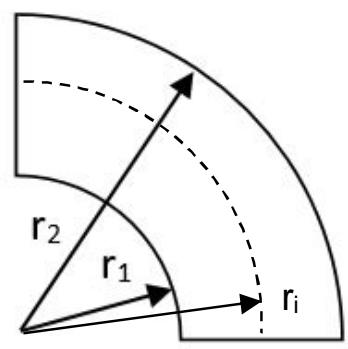

Figure 17. A quarter of annular

We focus on the various of temperatures in the circle $r_{i}$. In this study, we use $r_{i}=\left(r_{1}+r_{2}\right) / 2=0.15(\mathrm{~m})$. The coarse mesh is show in Figure 18, and h-Refinement technology was used to increase number of elements automatically, as show in Figure 19. 


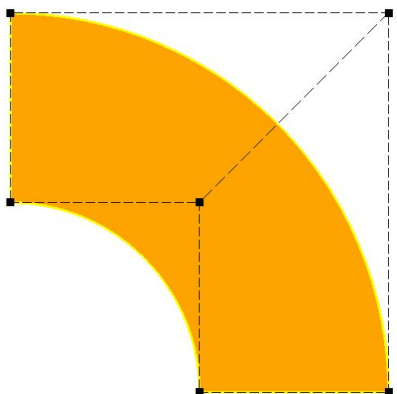

Figure 18. The coarse mesh of annular with only one element.

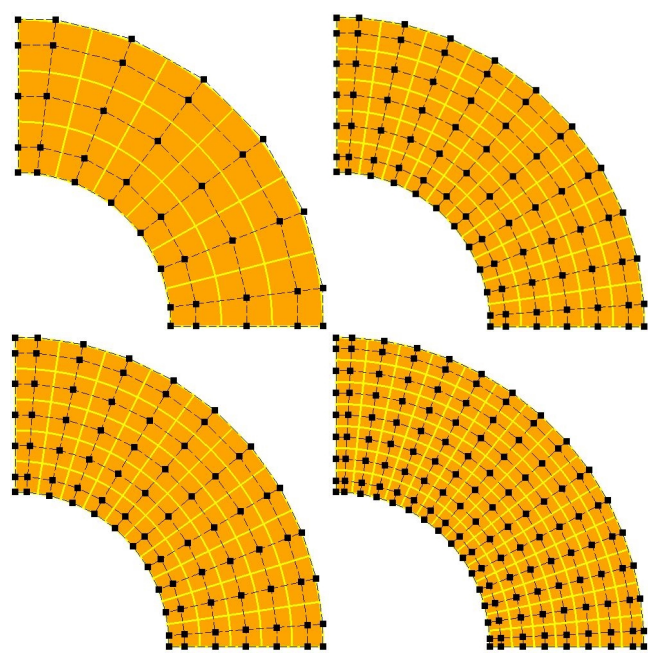

Figure 19. The refined mesh with $3 \times 6,5 \times 10$, $7 \times 14$ and $10 \times 20$

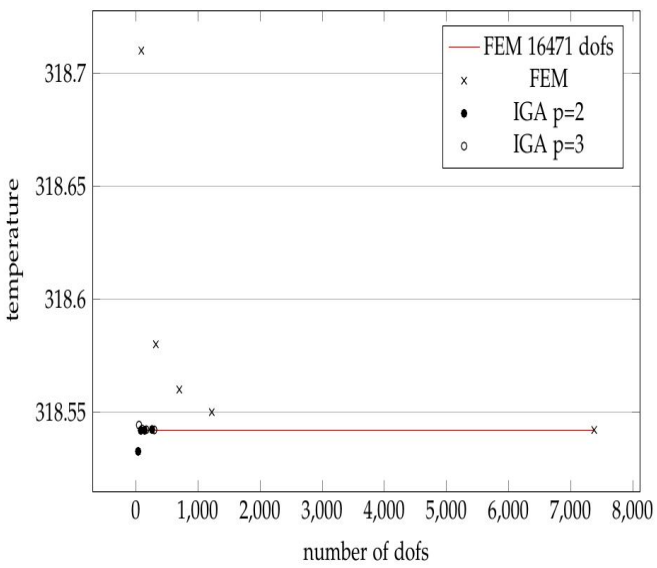

Figure 20. The convergency of temperature of point at the end of line ro, located at $r=0.15$ (m) and $\alpha=90^{\circ}$.
As

show

in

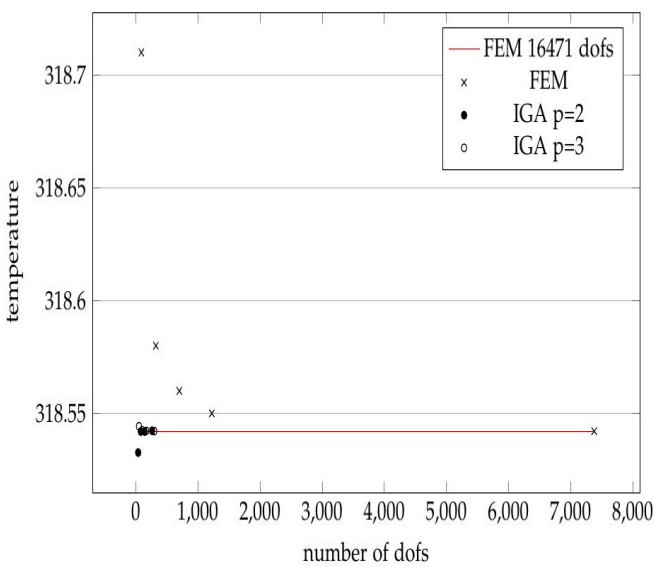

Figure 20, the convergence of IGA $(p=2)$ is better than FEM clearly. The result of FEM really accurate when number of dofs have increased more than 1000 dofs, while IGA just need more than 100 dofs.

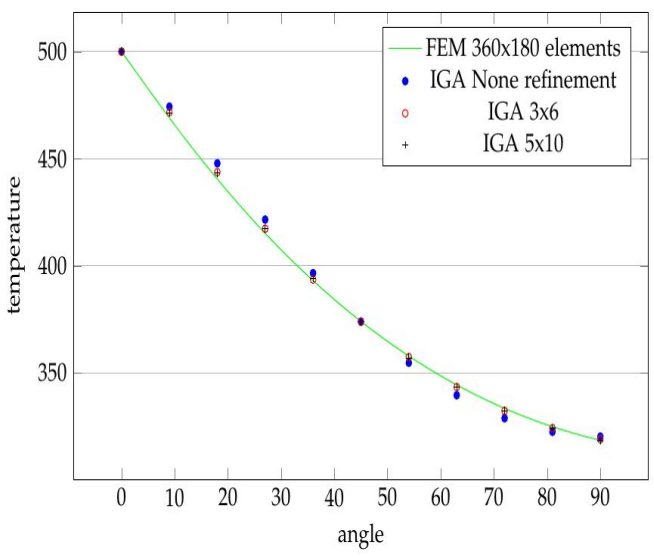

Figure 21. The convergency of temperature in line r0 for comparision of FEM with $360 \times 180$ elements.

We can recognize the various of temperatures is closed to FEM value while FEM's number DOFs is much higher. Eventhough, the result with only coarse mesh also be better.

The temperature contour is shown in Figure 22 and Figure 23. 


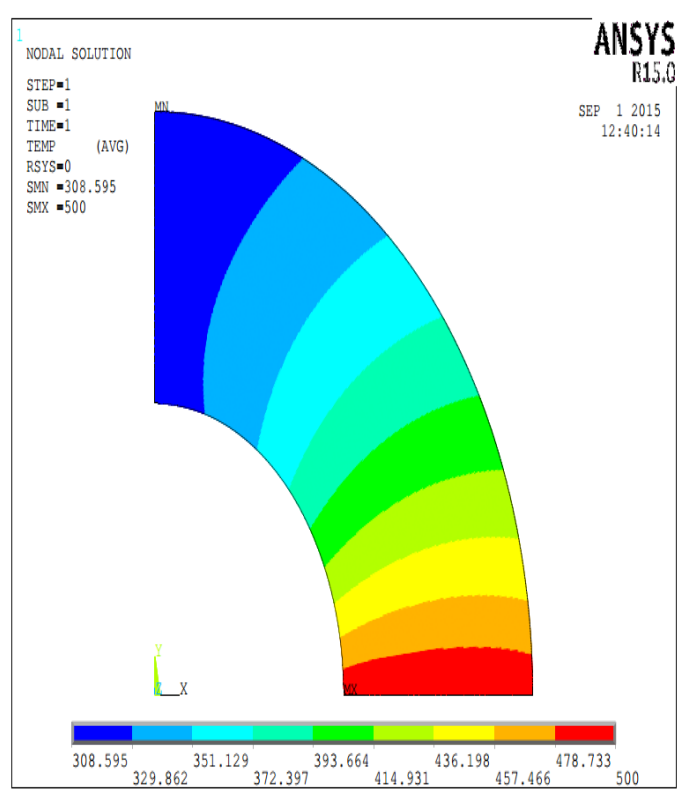

Figure 22. The contour of temperatures using FEM with 16471 dofs
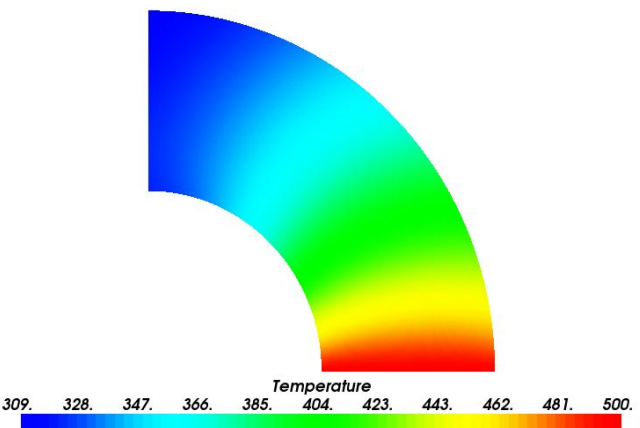

Figure 23. The contour of temperature using IGA with 231 dofs

\subsection{A practical problem}

This is a practice problem expressing the effect of IGA in heat transfer. The electrical technical advancement need to decrease the size of them, and a important problem is effect heat diffusion, or we can optimize the shape and dimension of heat sink. This section simply describe a few of problems listed above. That is the temperature distribution in fins of the heat sink to optimize it's profile. Consider to a heat sink is shown in Figure 24, it's made by
Aluminium alloy 6061, with the thermal conductivity values of $166 \mathrm{~W} / \mathrm{m} \cdot \mathrm{K}$. Assume the thermal conductivity value do not depend on the body temperature. The air through the heat sink which have the convection coefficient value of the fin of $25 \mathrm{~W} /\left(\mathrm{m}^{2} \mathrm{~K}\right)$.

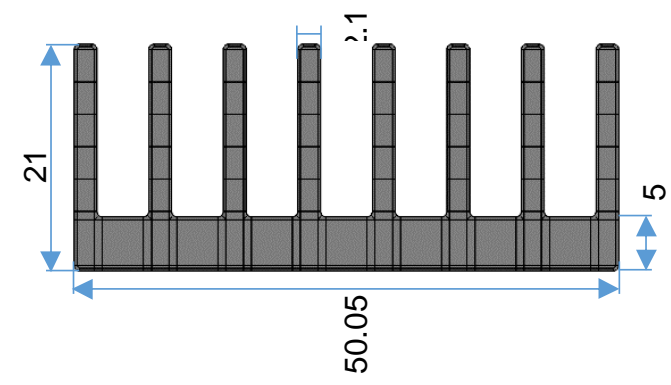

Figure 24. The heat sink model presented by NURBS surfaces

The NURBS surfaces of the heat sink is also shown in Figure 24. With that number of elements, the number of degrees of freedom is 1327, is shown in Figure 25. FEM solution with about 57000 degrees of freedoms is shown in Figure 26.

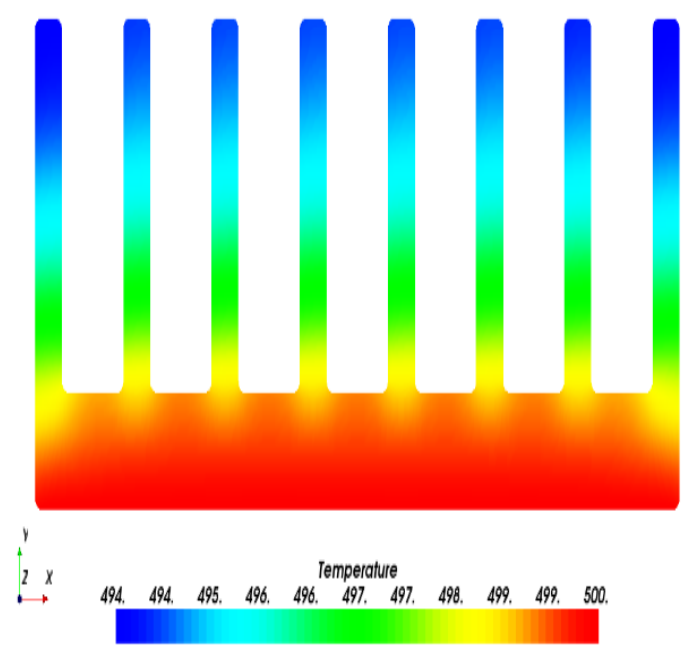

Figure 25. The temperature distribution of isogeometric analysis with about 1327 degrees of freedom 


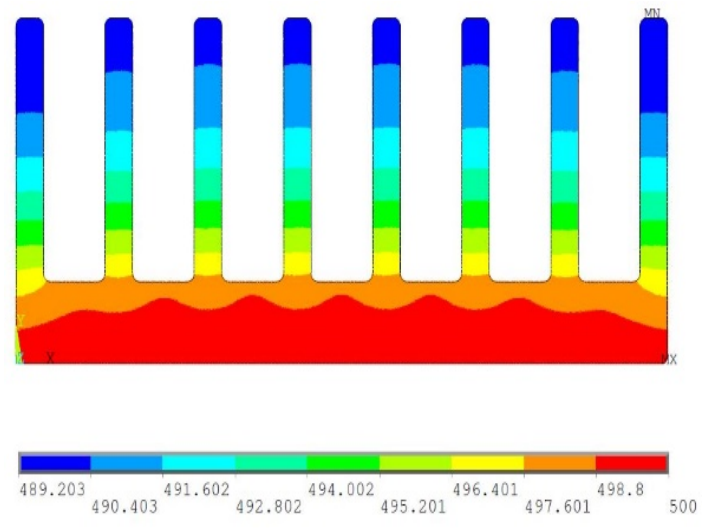

Figure 26. The temperature distribution of finite element method with about 57000 degrees of freedom

Consider to the temperature distribution on the second-fin of heatsink is shown in Figure 27. The FEM result is express by the red curve on the graph, and the temperature at the top is $489.96^{\circ} \mathrm{C}$. The cubic-IGA result is express by the blue curve and obtain $490.26^{\circ} \mathrm{C}$ at the top. The graph also show the result of both of two method are similar. Although, what is mentioning is that the degree of freedoms of IGA are less than FEM and it can decrease the memory capacity of computation. If we only interest in degree of freedoms in the second-fin, we can see an extremely different in number of them. In this study, the number of degree of freedoms of FEM are more than cubic-IGA 8 times, but in return, IGA base on high-order basis functions, and it can take a longer time. It can be a strong point and also a weak point.

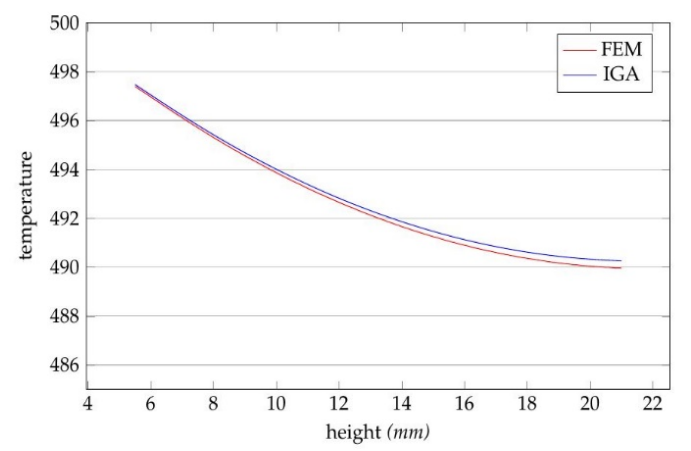

Figure 27. The temperature distribution in the fin

\section{CONCLUSION}

An isogeometric analysis approach for twodimensional static heat transfer problem is expressed above. Applying IGA to numerical problems lead significant effective results, as represent on above. More important that it can refine the mesh without the connection to the CAD geometry, it called h-refinement and $\mathrm{p}$ refinement and k-refinement, it is very convenient and makes the problem easier. Furthermore, IGA is base on high order basis functions, i.e., cubic basis functions are more often. Quartic basis functions have to take more time and the error decrease inappreciably, but they get a high accuracy in comparison with quadratic and cubic basis functions. Although, with industrial problems, where the accuracy is not necessary, FEM still gain advantages over. Therefore, IGA should be applied to problems that have complex geometries. It will decrease the errors at the compound curve, surface, it contributes to the exact results. IGA also have some disadvantages because it is still be developing. To make up the accuracy results, IGA is with regards to computational time to achieve convergence. A particular reason is high order basis functions must be spent more time to calculate. Summary, there is a basic of IGA application. We hope some problems mentioned above was enough to demonstrate the effect results of this analysis. 


\section{Dùng xấp xỉ phân tích đẳng hình học cho các bài toán truyền nhiệt ổn định hai chiều}

- Lê Tuấn Em

- Nguyễn Duy Khương

- Vũ Công Hòa

Trường Đại học Bách Khoa, ĐHQG-HCM

\section{TÓM TÁT:}

Mục đích của bài báo này là nghiên cứu áp dụng phân tích đẳng hình học cho bài toán tấm giải nhiệt qua cánh, một dạng bài toán truyền nhiệt ổn định hai chiều. Bằng cách sử dụng hàm dạng bậc cao như hàm NURBS, phân tích

Từ khóa: iga, truyền nhiệt, nurbs.

\section{REFERENCES}

[1]. S. T. N. M. B. Hassani, "Application of isogeometric analysis in structural shape optimization," Scientia Iranica, pp. 846-852, 2011.

[2]. O. a. C. J. Zienkiewicz, "Shape optimization and sequential linear programming," Optimum Structural Design, pp. 109-126, 1973.

[3]. U. K. D. K. A. R. Sabbir Hossain, "The Enhancement of Heat Transfer in a Circular Tube with Insert and without Insert by Using

đẳng hình học đạt tốc độ hội tụ cao khi so sánh với phương pháp phần tử hữu hạn truyền thống. Việc phát triển phương pháp này nhằm mục đích giảm khoảng cách giữa mô hình và mô phỏng và tăng tính liên tục cho mô hình lưới bài toán. the Finite Element Method," Procedia Engineering, vol. 105, pp. 81-88, 2015.

[4]. R. S. Davoud Mirzaei, Solving heat conduction problems by the Direct Meshless Local Petrov-Galerkin method, New York: Springer, 2013.

[5]. F. R. S.-Z. Edgar O. Reséndiz-Flores, "Twodimensional numerical simulation of heat transfer with moving heat source in welding using the Finite Pointset Method," International Journal of Heat and Mass 
Transfer, vol. 90, p. 239245, November 2015.

[6]. C. J. B. Y. Hughes TJR, Isogeometric analysis: CAD,finite elements, NURBS, exact geometry, and mesh refinement. Computer Methods in Applied Mechanics and Engineering, 2005.

[7]. J. P. Holman, Heat Transfer, McGraw-Hill.

[8]. W. T. Les Piegl, The NURBS Book, Springer, 1997.
[9]. P. N. K. N. S. Roland W.Lewis, Fundamentals of the Finite Element Method for Heat and Fluid Flow, John Wiley \& Sons Ltd, 2004.

[10].P. Dr. William S.Janna, Engineering Heat Transfer, CRC Press LLC, 2000.

[11].E. A. Al-Bahkali, "Finite Element Modeling for Thermal Stresses Developed in Riveted and Rivet-Bonded Joints," International Journal of Engineering \& Technology, vol. 11, pp. 106-112, December 2011. 